Winter Meeting - Joint meeting between the Nutrition Society and the Royal Society of Medicine, 11-12 December 2012, Dietary Strategies for the Management of Cardiovascular Risk

\title{
Eating behaviours in obese and lean women with polycystic ovary syndrome
}

\author{
Y. M. Jeanes ${ }^{1}$, J. Hodgson ${ }^{1}$, N. Saunders ${ }^{1}$, L. Gibson ${ }^{1}$ and K. Hart $^{2}$ \\ ${ }^{1}$ Health Sciences Research Centre, Roehampton University, London SW15 4JD and ${ }^{2}$ Department of Nutrition \& Metabolism, \\ Faculty of Health and Medical Sciences, University of Surrey, Guildford, GU2 7XH, UK
}

Polycystic ovary syndrome (PCOS) is the most prevalent endocrine disorder in women, affecting approximately $10 \%$ of women of reproductive age worldwide ${ }^{(1)}$. Prevalence of obesity is high in PCOS and is known to exacerbate the intrinsic feature of insulin resistance, which in turn worsens the metabolic and reproductive complications of the syndrome ${ }^{(2)}$. The associations between binge eating disorder and PCOS have not been robustly investigated but are key to improving the clinicians' understanding of the condition. Additionally food cravings have been anecdotally reported in women with PCOS (wPCOS) and postulated to influence body weight ${ }^{(3)}$. The aim of this observational study was to identify differences in food cravings and disordered eating behaviour between a group of obese and lean wPCOS in order to inform effective management strategies.

An online survey was constructed comprising items from several validated questionnaires including the Bulimic Investigatory Test (Edinburgh) (BITE), Three Factor Eating questionnaire (TFEQ) and Food Craving Questionnaire (FCQT) in addition to questions about weight management beliefs and strategies and basic subject characteristics. Volunteers were recruited via email advertisements and ethical approval was granted by the Universities Ethics Committees. Data was analysed using SPSS (v19). Independent t tests analyses were utilised to investigate differences in behavioural and demographic variables between weight groups.

Fifty wPCOS completed the survey $\left(n=34\right.$ obese and $n=16$ healthy weight); mean BMI was $35.0[3.7]$ and $22.5[1.4] \mathrm{kg} / \mathrm{m}^{2}(p<0.001)$. Obese wPCOS were significantly older than their lean counterparts (mean age: 32.8[5.3] v. 29.0[5.4]yrs, $p=0.02$ ). There was a significantly greater prevalence and severity of binge eating behaviours amongst obese wPCOS compared with lean wPCOS $(p=0.03$ and $p=0.04$ respectively). Similarly both uncontrolled eating and emotional eating were significantly more common amongst obese compared with lean wPCOS ( $p=0.03$ and $p=0.004$ respectively) and obese wPCOS scored significantly higher on two of the nine food craving sub scales: 'lack of control over eating' and 'emotions that may be experienced before or during cravings/eating' $(p=0.027$ and $p=0.011$ respectively). Compared with lean controls $(n=42)$ lean wPCOS $(n=16)$ demonstrated greater food craving $(p<0.05)$ and greater prevalence of binge eating behaviours $(p=0.03)$ indicating an influence of the syndrome independent of weight.

These findings provide an important insight into the nature and prevalence of comorbid disordered eating behaviours amongst women with PCOS. Such behaviours may be a contributor to and/ or function of the difficulties with weight management reported amongst women with PCOS and further research is needed to elucidate the direction of this effect.

1. March W, Moore VM, Willson, KJ, et al. (2010) Hum Reprod 25, 544-551.

2. Teede H, Deeks A and Moran L (2010) BMC Medicine 8, 41

3. Elsheikh M and Murphy C (2008) thefacts Polycystic Ovary Syndrome, Oxford, Oxford University Press. 